\title{
Kalman filter-based longitudinal phase-space reconstruction method for hadron machines
}

\author{
Diogo Alves $\odot^{*}$ and Kacper Lasocha $\odot^{\dagger}$ \\ CERN, CH-1211 Geneva 23, Switzerland
}

(Received 18 November 2020; accepted 24 June 2021; published 2 July 2021)

\begin{abstract}
This work proposes a novel longitudinal phase-space reconstruction method for hadron machines. The proposed method is based on a Kalman filter and can therefore provide real-time estimates of the phasespace reconstruction. The main input for the method is real-time measurements of the longitudinal bunch profile. Beam conditions in the LHC are used throughout this work as examples of the applicability and practical implementation of such a method. Longitudinal phase-space reconstructions obtained with the proposed method are compared with a tomographic-based technique using experimentally logged data from the LHC wall current monitors.
\end{abstract}

DOI: $10.1103 /$ PhysRevAccelBeams.24.072801

\section{INTRODUCTION}

The knowledge of the longitudinal phase space can provide valuable information on the quality of the beam including injection matching, capture performance, stability, time/phase spread, momentum/energy spread, longitudinal emittance, injection oscillations and filamentation. A tomographic-based reconstruction method coupled with a particle tracking code using longitudinal beam profile measurements has been proposed in [1]. Following this, other tomographybased implementations and system design proposals have been reported in different facilities [2-5].

We propose here a different approach based on a Kalman filter. The main advantages of this method are low computational load, sequential profile-by-profile processing and no need for prior trajectory calculations. It can be used in order to reconstruct the phase space, in real time, for arbitrarily long (i.e., unbounded) time intervals. In this work we start by deriving the unperturbed phase-space equations of motion for the case of hadron bunches, where typically the energy loss per turn is negligible. We then build a model for the evolution of the particle density in a polar discretization of the longitudinal phase space as well as a model that relates this density with the longitudinal bunch profile. Subsequently we show how these models can be incorporated in the context of a Kalman filter estimating the phase-space density using profile

\footnotetext{
*diogo.alves@cern.ch

Also at Institute of Physics, Jagiellonian University, Krakow, Poland.

Published by the American Physical Society under the terms of the Creative Commons Attribution 4.0 International license. Further distribution of this work must maintain attribution to the author(s) and the published article's title, journal citation, and DOI.
}

measurements as a sole input. Finally we present reconstruction results based on both real and simulated data, as well as a comparison with the tomography-based method. Conclusions and practical remarks are discussed at the end.

\section{SYNCHROTRON MOTION: TIME AND MOMENTUM OSCILLATIONS}

As shown in [6], for the typical case of hadron machines where the energy loss per turn is small compared to $q \hat{V}_{r f}$ (where $q$ denotes the particle charge and $\hat{V}_{r f}$ the peak rf voltage), the differential equation governing the rf phase difference $\Delta \phi_{r f_{i}}$ between bunch particle $i$ and the synchronous particle, as a function of time $t$, is given by

$$
\frac{d^{2} \Delta \phi_{r f_{i}}}{d t^{2}}+\Omega_{0}^{2} \sin \left(\Delta \phi_{r f_{i}}\right)=0
$$

where $\Omega_{0}$ is the nominal synchrotron frequency. Equation (1), valid for negligible deformations of the shape of the longitudinal potential well, has the form of the pendulum equation, for which a well-known approximate solution can be obtained from the traditional solution of the harmonic oscillator [7],

$$
\Delta \phi_{r f_{i}}=\widehat{\Delta \phi_{r f_{i}}} \cos \left(\Omega_{i} t+\phi_{i}\right),
$$

where ${\widehat{\Delta \phi_{r f}}}_{i}$ is the rf phase amplitude of synchrotron oscillations, $\phi_{i}$ is the initial phase and $\Omega_{i}=\Omega_{i}\left(\widehat{\Delta \phi_{r f_{i}}}\right)$ is the synchrotron frequency given by

$$
\Omega_{i}=\frac{\pi}{2 \mathcal{K}\left[\sin \left(\widehat{\frac{\Delta \phi_{r f i}}{2}}\right)\right]} \Omega_{0},
$$


where $\mathcal{K}(\cdot)$ is the complete elliptic integral of the first kind (page 590 of [8]). Both $\widehat{\Delta \phi_{r f}}$ and $\Omega_{i}$ are time invariant in the absence of nonconservative forces.

It was shown in [9] that for synchrotron oscillations with amplitudes satisfying $\widehat{\Delta \phi_{r f}} \lesssim 0.8 \pi$, which in the LHC corresponds to time oscillation amplitudes $\hat{\tau} \lesssim 1 \mathrm{~ns}$ (i.e., bunch lengths $\lesssim 2 \mathrm{~ns}$ ), Eqs. (2) and (3) provide a valid approximation to the general solution of Eq. (1). In addition, a second order approximation of Eq. (3) based on the MacLaurin series expansion, and which will prove useful later on, is given by Eq. (40) of [10]:

$$
\Omega_{i}=\left(1-\frac{{\widehat{\Delta \phi_{r f}}}_{i}^{2}}{16}\right) \Omega_{0} .
$$

Figure 1 shows the synchrotron frequency of particle $i$ as a function of its oscillation amplitude, as given by Eqs. (3) and (4). As can be seen, there is a good match between the approximate and the exact solutions for oscillation amplitudes $\widehat{\Delta \phi}_{r f_{i}} \lesssim 0.8 \pi$.

We shall consider the longitudinal phase $\phi_{L_{i}}(t)$ as the angular distance traveled by particle $i$ up to time $t$. Noting that $\Delta \phi_{r f_{i}}=h \times \Delta \phi_{L_{i}}$, where $h$ is the rf harmonic number ( $h=35640$ in the case of LHC) and $\Delta \phi_{L_{i}}$ is the longitudinal phase difference between particle $i$ and the synchronous particle, we can substitute it in Eq. (2) to write

$$
\Delta \phi_{L_{i}}=\widehat{\Delta \phi_{L_{i}}} \cos \left(\Omega_{i} t+\phi_{i}\right)
$$

Since by definition the longitudinal phase increment of the synchronous particle is exactly $\omega_{0} T_{0}=2 \pi$ per turn, where $\omega_{0}$ is the nominal angular revolution frequency and $T_{0}$ is the nominal revolution period, we have that the longitudinal phase of particle $i$ is given by

$$
\phi_{L_{i}}(t)=\omega_{0} t+\widehat{\Delta \phi_{L_{i}}} \cos \left(\Omega_{i} t+\phi_{i}\right)+\phi_{L},
$$

where $\phi_{L}$ is the initial phase of the bunch with respect to some longitudinal reference. Differentiating Eq. (6) we get the expression for the revolution frequency of particle $i$ as a function of time:

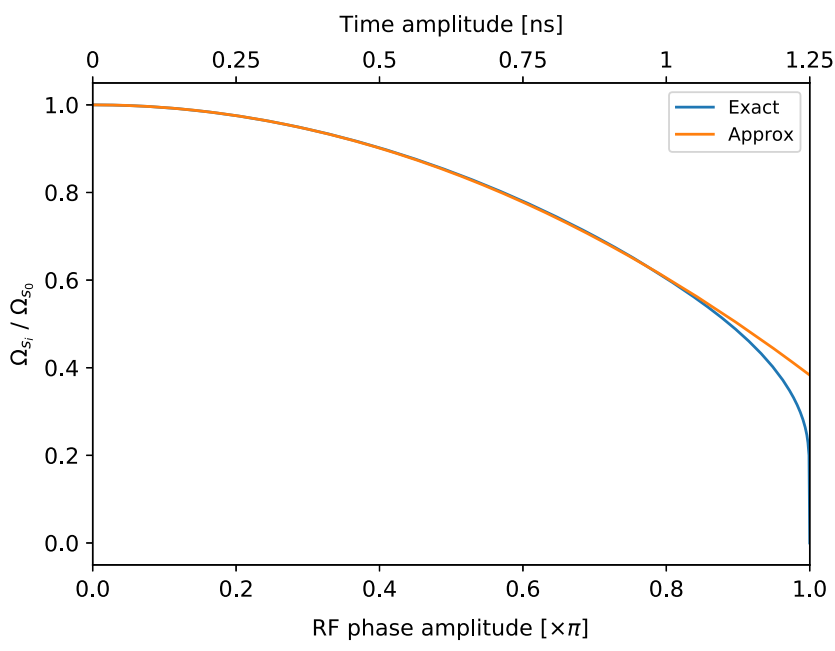

FIG. 1. Comparison between the exact [Eq. (3)] and approximate [Eq. (4)] expressions for the synchrotron frequency as a function of the oscillation amplitude.

$$
\omega_{L_{i}}(t)=\omega_{0}-\widehat{\Delta \omega_{L_{i}}} \sin \left(\Omega_{i} t+\phi_{i}\right)
$$

where $\widehat{\Delta \omega_{L_{i}}}=\Omega_{i} \widehat{\Delta \phi_{L_{i}}}$ is the amplitude of the oscillation in revolution frequency around its nominal value $\omega_{0}$.

We can now estimate the time difference between particle $i$ and the synchronous particle on a turn-by-turn basis. Let us start by calculating the Taylor series expansion of Eq. (6) in the vicinity of the instants $t=n T_{0}$, where $n$ represents an integer number of turns and $\tau_{n_{i}}$ represents the time difference between the moments when particle $i$ and the synchronous particle have the longitudinal phase $2 n \pi$. Without loss of generality, we can choose the arbitrary initial phase $\phi_{L}$ in Eq. (6) to be zero. Keeping only terms up to second order we can write

$$
\begin{aligned}
\phi_{L_{i}}\left(n T_{0}+\tau_{n_{i}}\right) \simeq & \phi_{L_{i}}\left(n T_{0}\right)+\tau_{n_{i}} \times \omega_{L_{i}}\left(n T_{0}\right) \\
& +\frac{\tau_{n_{i}}^{2}}{2} \times\left[\frac{d \omega_{L_{i}}}{d t}\right]_{t=n T_{0}}+\cdots=2 n \pi,
\end{aligned}
$$

which, after a bit of manipulation, becomes

$$
\tau_{n_{i}} \simeq \frac{-\widehat{\Delta \omega_{L_{i}}} \cos \left(n \Omega_{i} T_{0}+\phi_{i}\right)}{1-\widehat{\frac{\Delta \omega_{L_{i}} \Omega_{i}}{\omega_{0}}} \sin \left(n \Omega_{i} T_{0}+\phi_{i}\right)-\frac{\Omega_{i} \widehat{\Delta \omega_{L_{i}}}}{2 \omega_{0}} \tau_{n_{i}} \cos \left(n \Omega_{i} T_{0}+\phi_{i}\right)+\cdots} .
$$

From the previous we can conclude that if

$$
\epsilon_{k}=\left|\frac{\Omega_{i}^{k-1}}{k !} \frac{\widehat{\Delta \omega_{L_{i}}}}{\omega_{0}} \tau_{n_{i}}^{k-1}\right| \ll 1 \quad \text { for } k \in Z^{+},
$$

then Eq. (9) reduces to 


$$
\tau_{n_{i}} \simeq-\frac{\widehat{\Delta \omega_{L_{i}}}}{\omega_{o} \Omega_{i}} \cos \left(n \Omega_{i} T_{0}+\phi_{i}\right)
$$

In the case of hadron machines such as the LHC, for which we can use typical values at injection energy

$$
\begin{aligned}
& \Omega_{0} \approx 2 \pi \times 65 \mathrm{~Hz}, \\
&\left.\frac{\widehat{\Delta \omega_{L_{i}}}}{\omega_{0}}\right|_{1 \sigma} \approx 10^{-7}, \\
&|\tau|<\hat{\tau}_{L_{i}} \approx 1 \mathrm{~ns},
\end{aligned}
$$

we can clearly demonstrate that Eq. (10) holds.

Being able to write Eq. (10) is very interesting. First of all it confirms that the turn-by-turn time difference between particle $i$ and the synchronous particle is described by a simple harmonic motion, as stated in Eq. (16) of [11] without much justification. Furthermore, it immediately provides a relationship between the time amplitude of the synchrotron oscillations and the amplitude of the momentum deviation:

$$
\hat{\tau}_{L_{i}}=\frac{\widehat{\Delta \omega}_{L_{i}}}{\omega_{o} \Omega_{i}}=\frac{|\eta|}{\Omega_{i}} \frac{\widehat{\Delta p}_{i}}{p_{0}},
$$

where $\eta=1 / \gamma^{2}-\alpha$ represents the slip factor and $\alpha$ the momentum compaction factor. In the case of the LHC we have $\eta \approx-\alpha$ since the machine is operated well above transition energy.

In fact, Eq. (11) can also be found in Eq. (7) of [12] and in Eq. (9.70) of [13] in the context of low amplitude oscillations. What we demonstrate here is that, in the case of hadron machines, the expressions valid for low amplitude oscillations are still valid for maximum elongations of up to $80 \%$ of the length of the rf bucket, i.e., often within typical operating conditions.

Equation (11) is, of course, directly related to the bunch length. So, again for typical LHC parameters at injection energy, i.e.,

$$
\begin{aligned}
\Omega_{0} & \approx 2 \pi \times 65 \mathrm{~Hz}, \\
\eta & \approx-3.182 \times 10^{-4}, \\
\frac{\widehat{\Delta p_{i}}}{p_{0}} & \approx 4 \times 10^{-4},
\end{aligned}
$$

we get that

$$
\hat{\tau}_{L}^{4 \sigma} \approx 4 \times \frac{3.182 \times 10^{-4}}{2 \pi \times 65} \times 4 \times 10^{-4} \approx 1.25 \mathrm{~ns}
$$

which largely agrees with available bunch length measurements.
As previously discussed, an approximate solution for the pendulum equation [Eq. (1)] is defined by Eqs. (2) and (3). This means that each particle undergoes linear synchrotron motion (i.e., harmonic oscillations) at a frequency that depends on its amplitude of oscillation. The turn-by-turn momentum deviation of particle $i$ can therefore be written as in Sec. 9.2.1 of [13]:

$$
\Delta p_{n_{i}}=\widehat{\Delta p}_{i} \sin \left(n \Omega_{i} T_{0}+\phi_{i}\right),
$$

where the sign in front of $\widehat{\Delta p}_{i}$ reflects the assumption that the machine is operated above transition energy, again, certainly the case of the LHC. Momentum oscillations, described by Eq. (12), are therefore in phase quadrature with respect to the time oscillations, given by Eq. (10), and the knowledge of $\hat{\tau}_{i}$ and $\phi_{i}$ completely defines the motion.

Having established the relationships describing the motion in longitudinal phase space, we now proceed to propose an equivalent model in the case of a discretized phase space.

\section{DISCRETE PHASE-SPACE EVOLUTION MODEL}

We shall start by considering a polar phase-space discretization with angular resolution $\Delta \phi$ and radial resolution $\Delta \hat{\tau}$. Each point has discrete coordinates $\left(\phi_{i}, \hat{\tau}_{j}\right)$, for positive integers $i \in\left[1, N_{\phi}\right]$ and $j \in\left[1, N_{\hat{\tau}}\right]$, where $N_{\phi}$ and $N_{\hat{\tau}}$ are, respectively, the number of points of the angular grid and the number of points of the radial grid. An example representation of this grid is shown in Fig. 2.

To every point in this grid, we associate Cartesian coordinates $(\tau, v)$, related to the polar coordinates $(\phi, \hat{\tau})$ by

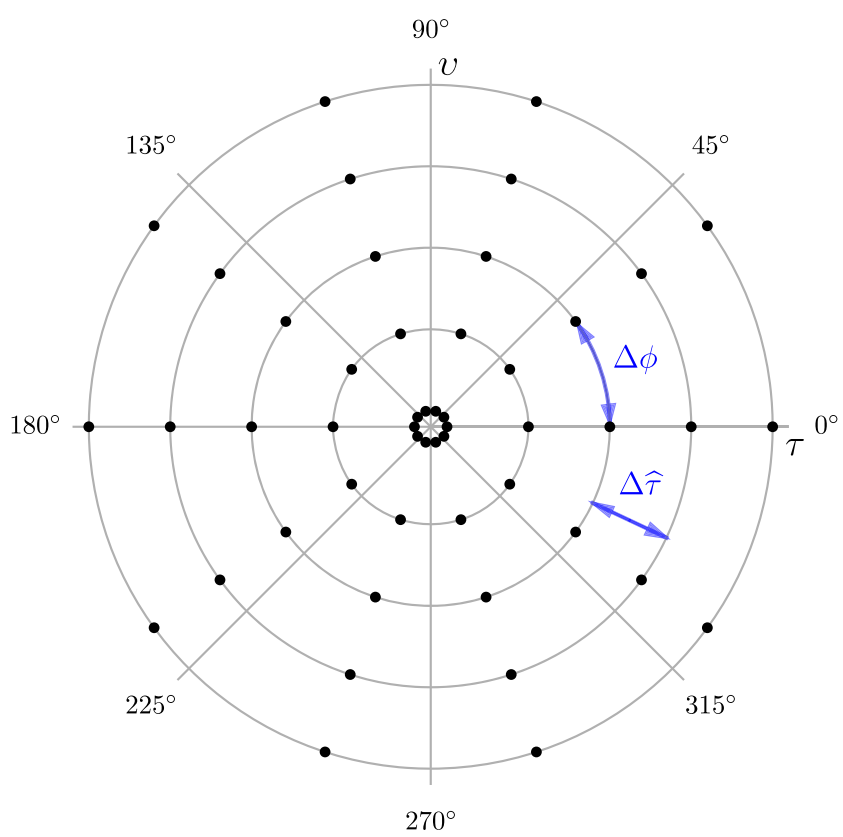

FIG. 2. Representation of a polar phase-space discretization. 


$$
\tau=\hat{\tau} \cos (\phi) \quad v=\hat{\tau} \sin (\phi) .
$$

The Jacobian of the transformation $(\phi, \hat{\tau}) \rightarrow(\tau, v)$ is $\hat{\tau}$ and the joint local probability densities of finding bunch particles at any time instant $t$ are related by (page 201 of [14])

$$
p_{\tau, v}(\tau, v, t)=\frac{p_{\phi, \hat{\tau}}(\phi, \hat{\tau}, t)}{\hat{\tau}} .
$$

Equation (14) will be useful in the next section.

Let us now consider a discrete time array $t_{k}$, with resolution $\Delta t$. From Eq. (10) we have that

$$
p_{\phi, \hat{\tau}}\left(\phi_{i}, \hat{\tau}_{j}, t_{k}\right)=p_{\phi, \hat{\tau}}\left(\phi_{i}-\Omega_{j} \Delta t, \hat{\tau}_{j}, t_{k-1}\right),
$$

where $\Omega_{j}$ is the synchrotron frequency of a particle with amplitude $\hat{\tau}_{j}$. This essentially means that, for a given amplitude $\hat{\tau}_{j}, p_{\phi, \hat{\tau}}\left(\phi_{i}, \hat{\tau}_{j}, t_{k}\right)$ rotates rigidly with synchrotron frequency $\Omega_{j} / 2 \pi$. Equation (15), determining the next state of the phase space based solely on the previous one, essentially defines a Markov process for the unperturbed evolution of a particle in longitudinal phase space. A complication arises from the fact that the angle $\phi_{i}-$ $\Omega_{j} \Delta t$ in general does not lie on a grid point. One solution is to linearly interpolate $p_{\phi, \hat{\tau}}\left(\phi_{i}-\Omega_{j} \Delta t, \hat{\tau}_{j}, t_{k-1}\right)$, between the two closest grid points of the same amplitude, using

$$
\begin{aligned}
& p_{\phi, \hat{\tau}}\left(\phi_{i}-\Omega_{j} \Delta t, \hat{\tau}_{j}, t_{k-1}\right) \\
& \simeq \alpha_{i m j} \times p_{\phi, \hat{\tau}}\left(\phi_{m}, \hat{\tau}_{j}, t_{k-1}\right) \\
& \quad+\left(1-\alpha_{i m j}\right) \times p_{\phi, \hat{\tau}}\left(\phi_{m-1}, \hat{\tau}_{j}, t_{k-1}\right),
\end{aligned}
$$

where $m$ is such that $\phi_{i}-\Omega_{j} \Delta t \in\left[\phi_{m-1}, \phi_{m}\right]$ and $\alpha_{i m j}=\left(\phi_{i}-\Omega_{j} \Delta t-\phi_{m-1}\right) / \Delta \phi$.

If we now simplify the notation by writing

$$
p_{i j}^{k}=p_{\phi, \hat{\tau}}\left(\phi_{i}, \hat{\tau}_{j}, t_{k}\right)
$$

and assume, for the sake of argument and without loss of generality, that $\Delta \phi>\Omega_{j} \Delta t, \forall j \in \mathbb{N}$ (i.e., $m=i$ ), we can express the time evolution of the probability density of every point in the phase-space grid by writing

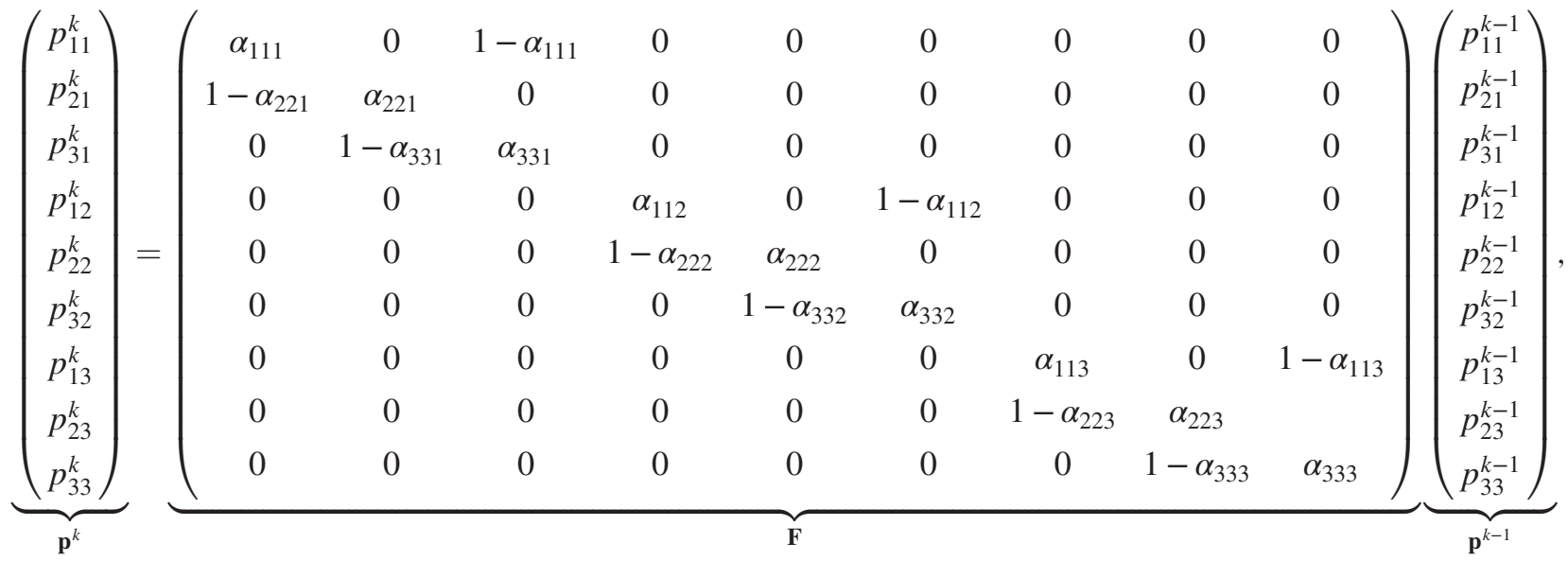

using $N_{\phi}=N_{\hat{\tau}}=3$ as an example. The dimension of $\mathbf{p}$ is $N_{\phi} N_{\hat{\tau}} \times 1$ and the dimension of $\mathbf{F}$ is $N_{\phi} N_{\hat{\tau}} \times N_{\phi} N_{\hat{\tau}}$.

It is thus possible to express the discrete time evolution of the local joint probability density, recursively, in matrix form.

\section{DISCRETE PHASE-SPACE MEASUREMENT MODEL}

The longitudinal bunch profile is the cumulative sum of the projections of all particles onto the horizontal $\tau$ axis. Let us define a discrete array $\tau_{i} \in\left(-N_{\hat{\tau}} \Delta \hat{\tau}, N_{\hat{\tau}} \Delta \hat{\tau}\right)$, having $N_{\tau}=2 N_{\hat{\tau}}-1$ entries spaced by $\Delta \tau=\Delta \hat{\tau}$, representing the time differences with respect to the synchronous particle, at which to calculate the longitudinal bunch profile. We can now define the relationship between the probability density in the discretized phase space and the longitudinal bunch profile $\lambda(\tau)$. For this we will refer to Fig. 3 and note that the relative number of particles (also commonly referred to as line density) measured at horizontal grid point $\left(\tau=\tau_{2}, v=0\right)$ can be approximated by

$$
\begin{aligned}
\lambda\left(\tau_{2}\right) \approx & {\left[p_{\tau, v}\left(P_{1}^{T}\right)+p_{\tau, v}\left(P_{1}^{B}\right)\right] l_{1} \delta \tau } \\
& +\left[p_{\tau, v}\left(P_{2}^{T}\right)+p_{\tau, v}\left(P_{2}^{B}\right)\right] l_{2} \delta \tau
\end{aligned}
$$

where the labels $T$ and $B$ denote, respectively, the $T o p$ and Bottom points. The probability densities in the previous expression can be interpolated by 


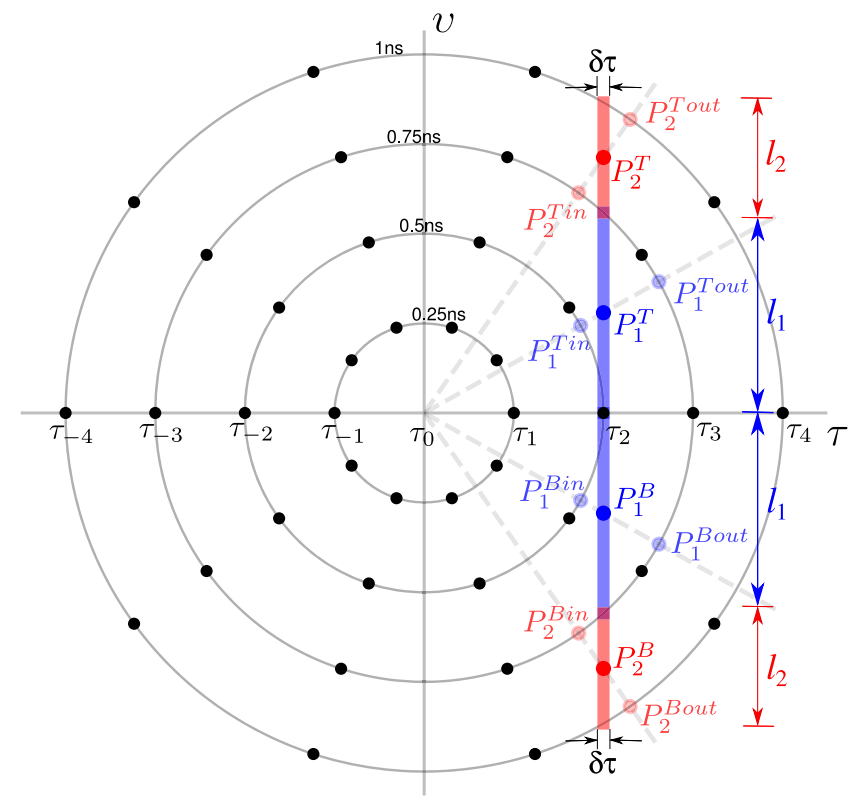

FIG. 3. Auxiliary figure supporting the explanation on how to express the longitudinal bunch profile at $\left(\tau=\tau_{2}, v=0\right)$ as a function of the local joint probability densities.

$$
\begin{aligned}
p_{\tau, v}\left(P_{n}^{T / B}\right) \approx & \frac{\hat{\tau}\left(P_{n}^{T / B, \text { out }}\right)-\hat{\tau}\left(P_{n}^{T / B}\right)}{\hat{\tau}\left(P_{n}^{T / B, \text { out }}\right)-\hat{\tau}\left(P_{n}^{T / B, \text { in }}\right)} p_{\tau, v}\left(P_{n}^{T / B, \text { in }}\right) \\
& +\frac{\hat{\tau}\left(P_{n}^{T / B}\right)-\hat{\tau}\left(P_{n}^{T / B, \text { in }}\right)}{\hat{\tau}\left(P_{n}^{T / B, \text { out }}\right)-\hat{\tau}\left(P_{n}^{T / B, \text { in }}\right)} p_{\tau, v}\left(P_{n}^{T / B, \text { out }}\right),
\end{aligned}
$$

where $\hat{\tau}(P)$ denotes the synchrotron amplitude at point $P$ and the labels in and out denote, respectively, the inner and outer points. From Eq. (14) we have that

$$
p_{\tau, v}\left(P_{n}^{T / B, \text { in } / \text { out }}\right)=\frac{p_{\phi, \hat{\imath}}\left(P_{n}^{T / B, \text { in } / \text { out }}\right)}{\hat{\tau}},
$$

and finally from Eq. (16) we obtain $p_{\phi, \hat{\tau}}\left(P_{n}^{T / B \text {,in/out }}\right)$.

In summary, the procedure to calculate $\lambda(\tau)$ is the following: from Eq. (16) we can calculate $p_{\phi, \hat{\tau}}\left(P_{n}^{T / B \text {,in/out }}\right)$, then, from Eq. (20) we calculate $p_{\tau, v}\left(P_{n}^{T / B \text {,in/out }}\right)$, this enables us to calculate $p_{\tau, v}\left(P_{n}^{T / B}\right)$ from Eq. (19) and finally get $\lambda(\tau)$ from Eq. (18), where $l_{1}$ and $l_{2}$ are obtained from direct geometric considerations and $\delta \tau$ is in practice an arbitrary constant whose value is independent of the measurement point and thus does not affect the shape of the profile.

Using the procedure described in this section for a predefined polar grid in phase space, the overall longitudinal bunch profile can be expressed as a sum of linear combinations of probability densities via

$$
\lambda^{k}=\mathbf{H} \mathbf{p}^{k},
$$

where $\mathbf{H}$ is calculated almost strictly from geometric principles. The bunch profile vector $\lambda$ has dimensions $N_{\tau} \times 1$ and the matrix $\mathbf{H}$ has dimensions $N_{\tau} \times N_{\phi} N_{\hat{\tau}}$.

\section{KALMAN FILTER IMPLEMENTATION}

The Kalman filter (KF) [15] is an estimator which incorporates information from both theoretical models and experimental measurements in order to provide optimal estimates of the state of a system. The Kalman filter works in two stages. In the first stage the filter uses the theoretical model to predict the evolution of the state of the system (prior state estimate). In the second stage this prediction is corrected by incorporating the experimental measurements (posterior state estimate). The Kalman filter is essentially applicable to Markov processes whose state evolution can be described by a linear model and whose measurements can be related to the system state also via a linear model.

The two fundamental expressions required for the use of the Kalman filter have already been derived. These are the process evolution model, Eq. (17), and the measurement model, Eq. (21).

The overall idea of the Kalman filter is evident from the following expressions:

$$
\begin{aligned}
\mathbf{p}^{k \mid k-1} & =\mathbf{F} \mathbf{p}^{k-1 \mid k-1}, \\
\lambda^{k \mid k-1} & =\mathbf{H p}^{k \mid k-1},
\end{aligned}
$$

and

$$
\mathbf{p}^{k \mid k}=\mathbf{p}^{k \mid k-1}+\mathbf{K}^{k}\left(\lambda_{\exp }^{k}-\lambda^{k \mid k-1}\right) .
$$

All $k \mid k-1$ indexes refer to a prediction (prior) and all $k \mid k$ indexes refer to a correction (posterior). $\lambda_{\exp }^{k}$ denotes the experimental measurements of the longitudinal beam profile taken at time index $k, \lambda^{k \mid k-1}$ are the predicted measurements, $\mathbf{K}$ is the Kalman filter gain (which will be discussed shortly) and $\mathbf{p}^{k \mid k}$ is the final estimation of the phase-space density distribution for discrete time index $k$.

In order to apply the set of Eqs. (22) to (24) we need to calculate the Kalman gain $\mathbf{K}$. The gain can be obtained for every time index $k$ using the following set of expressions:

$$
\begin{gathered}
\mathbf{P}^{k \mid k-1}=\mathbf{F P}^{k-1 \mid k-1} \mathbf{F}^{T}+\mathbf{Q}, \\
\mathbf{K}^{k}=\mathbf{P}^{k \mid k-1} \mathbf{H}^{T}\left(\mathbf{H} \mathbf{P}^{k \mid k-1} \mathbf{H}^{T}+\mathbf{R}\right)^{-1},
\end{gathered}
$$

and

$$
\mathbf{P}^{k \mid k}=\left(\mathbf{I}-\mathbf{K}^{k} \mathbf{H}\right) \mathbf{P}^{k \mid k-1},
$$

where $\mathbf{Q}$ is the process covariance matrix, $\mathbf{R}$ is the measurement covariance matrix and $\mathbf{P}$ is the covariance matrix of the combined estimates. Matrix $\mathbf{Q}$ represents the 
joint variability of the process and can be chosen arbitrarily, while $\mathbf{R}$ represents the joint variability of the measurement. For simplicity we assume that both matrices are of the form

$$
\mathbf{Q}=q \mathbf{I}_{Q}
$$

and

$$
\mathbf{R}=r \mathbf{I}_{R},
$$

where $\mathbf{I}_{Q}$ and $\mathbf{I}_{R}$ are, respectively, the $N_{\phi} N_{\hat{\imath}} \times N_{\phi} N_{\hat{\imath}}$ and $N_{\tau} \times N_{\tau}$ identity matrices, meaning that neither of them exhibits internal correlations and that all variances are uniform. Essentially, small values of $\mathbf{K}$ favor the prediction stage (i.e., enhances the weight given to the process model) while large values of $\mathbf{K}$ favor the correction stage (i.e., enhances the weight given to the experimental measurements). This balance depends on the relative magnitude of the elements in the $\mathbf{Q}$ matrix with respect to the ones in the $\mathbf{R}$ matrix. The shape of matrices $\mathbf{Q}$ and $\mathbf{R}$ does not restrict the potential solution to a specific case, but might however impact the convergence. In Eqs. (28) and (29) we have chosen the simplest example, which may be refined based on the properties of the measurement system, adopted Kalman filter model and requirements of the desired phasespace reconstruction.

We can see from Eqs. (25)-(27) that the Kalman filter gain $\mathbf{K}$ is independent of measurements and state estimates. In fact, $\mathbf{K}$ depends only on $\mathbf{F}, \mathbf{H}, \mathbf{Q}$ and $\mathbf{R}$, which are all stationary matrices. This means that, in principle, $\mathbf{K}$ can be precalculated by recursively evaluating Eqs. (25) to (27) and verifying that it converges to a steady-state value $\mathbf{K}_{\infty}$. Alternatively, one can substitute $\mathbf{P}^{k \mid k-1}$, from Eq. (25), into Eq. (26) to obtain the discrete algebraic Riccati equation which, in case of convergence, can also be used to calculate $\mathbf{K}_{\infty}$ (page 194 of [16]). Even in case of convergence to a fixed value $\mathbf{K}_{\infty}$, the resulting filter is only stable if the absolute value of each of the eigenvalues of $\left(\mathbf{I}-\mathbf{K}_{\infty} \mathbf{H}\right) \mathbf{F}$ is less than 1 (page 196 of [16]). For the Kalman filter parameters used in the examples presented in the next section, we have verified that the recursive evaluation of Eqs. (25)-(27) leads to a stationary value $\mathbf{K}_{\infty}$ and that the resulting filter is indeed stable.

In terms of computational load, and since the Kalman gain can be precomputed, this method is extremely interesting since it requires no more than three matrix-vector multiplications and two vector sums [i.e., Eqs. (22) to (24)] on each iteration to provide a phase-space estimate in polar coordinates. Furthermore, it does not require the real-time evaluation of trigonometric or other complex functions which are computationally expensive.

An important aspect to note is that we have defined $p_{\phi, \hat{\tau}}(\phi, \hat{\tau}, t)$ as a probability density. As such we have to guarantee that $p_{\phi, \hat{\tau}}\left(\phi_{i}, \hat{\tau}_{j}, t_{k}\right)>0, \forall i, j, k$, which is the same as saying that not a single element of $\mathbf{p}^{k \mid k}$, calculated using Eq. (24), can be negative. In order to satisfy this we need to use a version of the Kalman filter that can handle state constraints. We have therefore implemented the approach described in Sec. 2.4 of [17] with hardly an impact in terms of computation time with respect to the classical unconstrained Kalman filter.

\section{RESULTS}

In order to test the feasibility of this method as an online phase-space reconstruction tool, we have tested it using turn-by-turn LHC wall current monitor (WCM) longitudinal bunch profile measurements as an input. The chosen dataset consists of 398 profiles acquired at a rate of 40 Gsps (i.e., approximately 100 points per profile), covers the first turns after injection and shows strong oscillations due to an initial phase mismatch between the bunch and the rf waveform. For this dataset, we compare the results of the Kalman-based reconstruction with the ones obtained using the standard tomography-based reconstruction method described in [5].

The Kalman filter was configured with $N_{\phi}=144$, which corresponds to $\Delta \phi=2.5^{\circ}$ for the angular grid, $N_{\hat{\imath}}=40$ and $N_{\tau}=79$ (which corresponds to $\Delta \hat{\tau}=\Delta \tau=12.5 \mathrm{ps}$ ) since, as we have seen before, this approach is only valid for elongations up to $80 \%$ of the bucket length. As a consequence, the first ten and last eleven points of each profile (with negligible number of particles compared to the center of the bucket) are ignored. From the rf settings at the time of injection we know that the synchrotron frequency is approximately $45.82 \mathrm{~Hz}$ which, in the case of the LHC, corresponds to a synchrotron tune of $4.07 \times 10^{-3}$. This means that, during the aforementioned 398 turns, we observe approximately 1.5 synchrotron periods. Regarding matrices $\mathbf{Q}$ and $\mathbf{R}$, respectively given by Eqs. (28) and (29), we have made $q=r=1$, i.e., comparable weight is given to both the theoretical model and the profile measurements. Finally, we have used uniform distributions for both the synchrotron phase and amplitudes as an initial guess of the phase-space configuration.

As previously seen, the Kalman filter estimates the phase-space density in polar coordinates, i.e., it estimates $p_{\phi, \hat{\tau}}(\phi, \hat{\tau})$. In order to express this density in the $\left(\tau, \Delta p / p_{0}\right)$ coordinate system, we can make use of the transformation $(\phi, \hat{\tau}) \rightarrow\left(\tau, \Delta p / p_{0}\right)$ defined by

$$
\tau=\hat{\tau} \cos (\phi), \quad \frac{\Delta p}{p_{0}}=-\frac{\Omega(\hat{\tau}) \hat{\tau}}{|\eta|} \sin (\phi) .
$$

The choice of signs in the above equation is the result of the negative slip factor for machines operating above transition energy. This implies the clockwise rotation of the phase space. Adopting the sign convention opposite to Eqs. (10) and (11) has no impact on the model and is consistent with Eq. (13). 
If we now use Eq. (4) to express $\Omega(\hat{\tau})$ and note that ${\widehat{\Delta \phi_{r f i}}}_{i}=h \omega_{0} \hat{\tau}_{i}$ where $h$ is the rf harmonic number, we can show (page 201 of [14]) that the joint probability densities, $p_{\phi, \hat{\tau}}(\phi, \hat{\tau})$ and $p_{\tau, \frac{\Delta p}{p_{0}}}\left(\tau, \frac{\Delta p}{p_{0}}\right)$, are related by

$$
p_{\tau, \frac{\Delta p}{p_{0}}}\left(\tau, \frac{\Delta p}{p_{0}}\right)=\frac{p_{\phi, \hat{\tau}}(\phi, \hat{\tau})}{\frac{\Omega_{0} \hat{\tau}}{|\eta|}\left[1-\frac{h^{2} \omega_{0}^{2} \hat{\tau}^{2}}{8}\left(\sin ^{2}(\phi)-\frac{1}{2}\right)\right]},
$$

where $\phi$ and $\hat{\tau}$ are given by the inverse transform of Eq. (30). Essentially, Eq. (31) defines a stationary map which can be precomputed, and that has to be applied to the Kalman filter estimate $p_{\phi, \hat{\tau}}(\phi, \hat{\tau})$ in order to convert the phase-space density to the more relevant $\left(\tau, \Delta p / p_{0}\right)$ set of coordinates.

The results from both the Kalman filter phase-space reconstruction and the standard tomography-based method are shown in Fig. 4. Since the adopted synchrotron motion model is only valid for particles within the central $80 \%$ of the bucket, particles closer to the edge of the rf bucket are not present in the KF estimate.

While the standard tomography method uses the full dataset of 398 profiles (iterating over all of them a predefined number of times) in order to calculate the phase-space evolution turn by turn, the Kalman filter process is recursive and provides an estimate in real time, having only the present profile as an input at each step. A consequence of this fact is that the filter needs some time to converge to the true phase space. We can observe the reminiscences of this process in the form of artifacts present
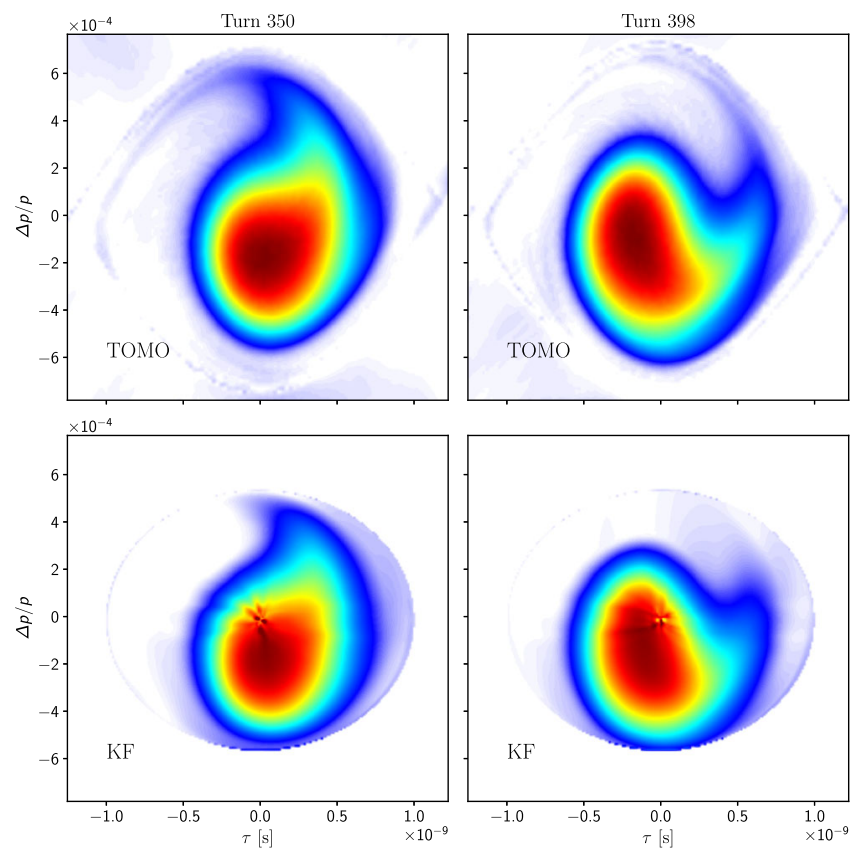

FIG. 4. Comparison between the results of the standard tomography phase-space reconstruction (TOMO) and the Kalman filter-based reconstruction $(\mathrm{KF})$ in the presence of injection oscillations.
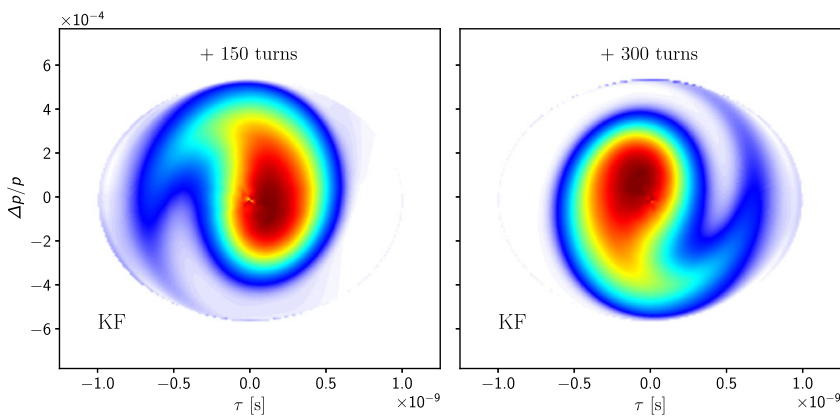

FIG. 5. Kalman filter phase-space reconstruction of the additional turns. Input longitudinal profiles based on the Monte Carlo simulation.

in the center of the phase space. As we do not have profile measurements beyond the 398th turn for this fill, we support this statement with two simulations. First, following the distribution given by the tomography based phasespace reconstruction for turn 398, we draw a Monte Carlo sample of $5 \times 10^{5}$ particles and track their evolution, which is assumed to follow the synchrotron motion described in Sec. II. Projecting such an ensemble on the $\tau$ axis we obtain further longitudinal bunch profiles, which can be analyzed with the KF method. In phase spaces obtained in such a way after processing additional 150 and 300 turns the impact of the central artifact is progressively reduced, as can be seen in Fig. 5.

An additional verification comes with analysis of the early steps of the filter convergence. In order to focus only on this aspect, a well-centered bunch with a stationary longitudinal bunch profile was simulated. In Fig. 6 we can
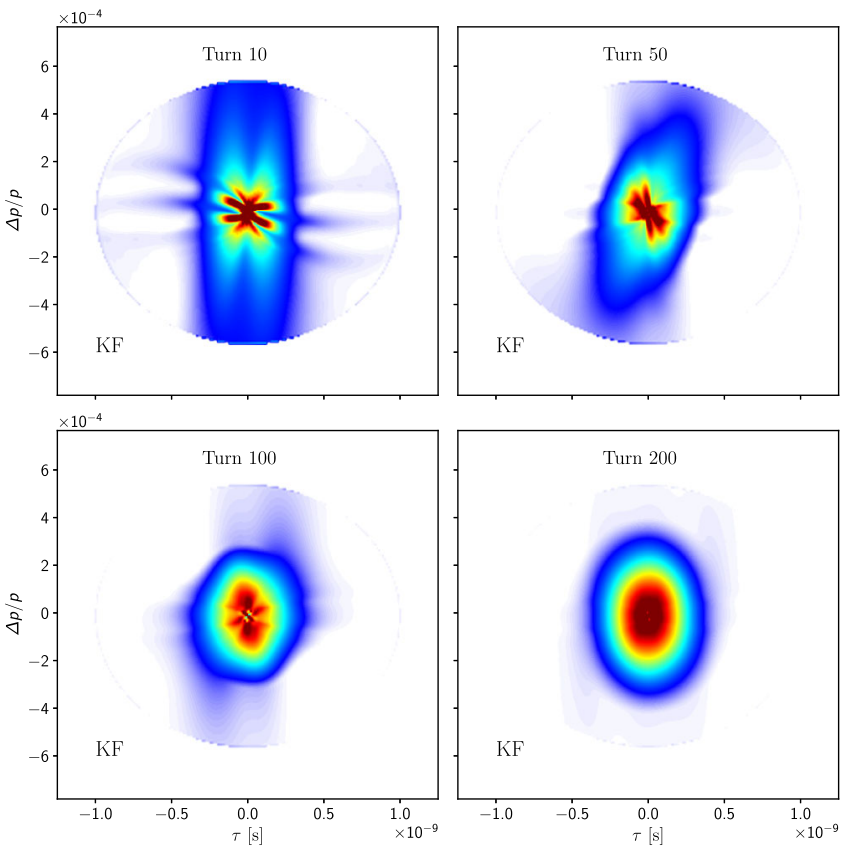

FIG. 6. Early steps of the Kalman filter phase-space reconstruction in the case of the well-centered stationary bunch. 
see that the phase-space estimate gradually improves and requires, in this particular case, approximately 200 turns for the full convergence where the central artifact is no longer visible.

\section{APPLICABILITY TO OTHER BEAM CONDITIONS}

All considerations up to this point, including the validation of the synchrotron motion approximation as well as the construction of the state-transition matrix $\mathbf{F}$ and observation matrix $\mathbf{H}$ were made under the assumption of LHC-like parameters in stationary conditions. In this section we would like to address what is the range of applicability of the Kalman filter-based longitudinal phasespace reconstruction method, and what changes may be required.

The state-transition matrix $\mathbf{F}$ has been constructed in Sec. III under the assumption that the energy loss by a particle in a single turn is small relative to its total energy and that particles are not present near the boundaries of the rf bucket. In addition, the rf system is, or is equivalent to, a single-cavity stationary system. This, as discussed in Sec. II, results in a sinusoidal synchrotron motion, with an amplitude-dependent synchrotron frequency. Such an assumption is valid for the LHC, as well as for other hadron machines, such as RHIC [18] (when operating with a single rf system) and J-PARC main ring [19]. In these cases all the derivations presented in this paper remain valid and we should only change the relevant machine-specific parameters (nominal synchrotron frequency, slip factor and rf bucket length).

In the case of high energy losses, as in lepton machines (FCC-ee, CEPC), multiharmonic rf systems as in the SPS [20], nonsinusoidal rf systems [21], or in the presence of particles near the boundaries of the rf bucket, the results of Sec. II are not valid, and, as a consequence, the statetransition matrix $\mathbf{F}$ would be different from the one presented in Sec. III. Still, such a matrix may be precomputed based on the knowledge of the rf system and phasespace evolution model, including e.g. impedance, radiation losses and space charge. The limitation being that the underlying process cannot be chaotic, like in certain double rf system configurations [22].

\section{CONCLUSIONS AND PRACTICAL CONSIDERATIONS}

A new approach for the reconstruction of the longitudinal phase-space based on profile measurements, and with direct application to hadron machines, has been developed. The method, based on a Kalman filter, strongly depends on the adopted model of the synchrotron motion. Examples assuming sinusoidal synchrotron motion, with an amplitude-dependent frequency were shown to be valid for bunch lengths of up to about $80 \%$ of the single-harmonic rf bucket length. Although it represents an undeniable limitation of the model, not being able to cope with larger oscillation amplitudes, for many hadron machines such as the LHC, this covers the overwhelming majority of operating time and scenarios. The method has been tested using longitudinal bunch profiles from WCMs in the LHC and compared with the standard phase-space tomography, showing good agreement.

The method, with the synchrotron motion model presented in this paper, requires the knowledge of the nominal synchrotron frequency, which can be obtained from Eq. (9.35) of [13] knowing the nominal momentum, the peak rf voltage and the cosine of the synchronous phase. For example, in the LHC, even during acceleration, the synchronous phase is always $<5^{\circ}$. This means that its cosine is always close to one. Since the values of the nominal momentum and maximum rf voltage are available in real time, this does not represent a problem. Impedance effects leading to significant shifts of the synchronous phase and of the synchrotron frequency are not taken into account and are out of the scope of this paper. Alternatively, the nominal synchrotron frequency can be obtained from fitting the Schottky spectrum using, for example, the method described in [9]. In the case of storage ring colliders such as the LHC, the synchrotron frequency changes only during acceleration remaining otherwise stationary.

An additional requirement of this method is that the profile measurements need to be synchronous with the rf waveform, so that the center of the bucket is precisely known. Such a synchronization in the case of LHC is not difficult, as profile measurements are triggered with a jitter of approximately $\pm 25 \mathrm{ps}$ with respect to the rf clock.

In Sec. VI we have used turn-by-turn profile measurements. In this particular case, this corresponds to acquiring approximately 245 profile measurements per synchrotron period. Such a high profile acquisition frequency is not strictly necessary and one could consider reducing the frequency of this update to, for example, 20 profile measurements per nominal synchrotron period. This would help reduce the computational demand of a potential realtime implementation. Similarly, one could also consider increasing the spacing of the angular grid, thus reducing matrix sizes in the Kalman filter implementation. Doing so would however increase the discretization error, degrading the quality of the reconstruction.

The validity conditions of this method were discussed. Impedance effects leading to a significant deformation of the potential well within the bunch length region would modify the $\mathbf{F}$ matrix and are not taken into account. Thorough studies on the behavior of the method under such conditions can be performed and provide an interesting research topic for the future. In principle, any perturbations occurring at timescales much longer than the profile update period should be taken into account by the correction term of the filter depending on its bandwidth (i.e., the 
values of $r$ and $q$ ). Likewise, also the possible propagation of discretization errors should be mitigated. Furthermore, zero-mean perturbations occurring at timescales much smaller than the profile update period should also, in theory, have no impact on the filter performance. Problems are expected to arise from nonzero-mean perturbations occurring at timescales smaller or comparable to the profile update period. Still, all deviations from the undisturbed synchrotron motion will in principle have an impact on the measured profiles and will therefore be taken into account by the filter.

When it comes to using experimental beam profile measurements, we have not considered the possibility of the bunch spectrum having relevant frequency content beyond the bandwidth of the instrument. However, this can be taken into account by modifying the measurement matrix $\mathbf{H}$ in order to approximate the bandwidth of the predicted profile to the experimentally obtained one.

We plan to apply this method directly on the acquired turn-by-turn longitudinal profiles measured during the next LHC run (2022-2024) and assess its performance. In addition, we also plan to continue developing this method not only to take into account impedance effects but also to estimate their contribution. Such a development will likely make use of one of the nonlinear implementations of the Kalman filter and will be a natural evolution of the present work.

\section{ACKNOWLEDGMENTS}

The authors would like to thank O.R. Jones and T. Lefèvre for their relevant suggestions and express their gratitude towards T. Argyropoulos for providing WCM profile measurements, the tomographic reconstructions used for the comparisons and for the numerous discussions.

[1] S. Hancock, M. Lindroos, E. McIntosh, and M. Metcalf, Tomographic measurements of longitudinal phase space density, Comput. Phys. Commun. 118, 61 (1999).

[2] C. Montag et al., Longitudinal phase space tomography in RHIC, in Proceedings of the 8th European Particle Accelerator Conference, Paris, 2002 (EPS-IGA and CERN, Geneva, 2002), p. 6.

[3] H. Loos, P. R. Bolton, J. E. Clendenin, D. H. Dowell, S. M. Gierman, C. G. Limborg, J. F. Schmerge, T. V. Shaftan, and B. Sheehy, Longitudinal phase space tomography at the SLAC gun test facility and the BNL DUV-FEL, Nucl. Instrum. Methods Phys. Res., Sect. A 528, 189 (2004).

[4] D. Malyutin, M. Gross, I. Isaev, M. Khojoyan, G. Kourkafas, M. Krasilnikov, B. Marchetti, M. Otevrel, F. Stephan, and G. Vashchenko, Longitudinal phase space tomography using a booster cavity at pitz, Nucl. Instrum. Methods Phys. Res., Sect. A 871, 105 (2017).

[5] C. H. Grindheim and S. Albright, Longitudinal phase space tomography version 3, CERN Accelerator Notes No. 2021-0004, 2021.

[6] M.H. Blewett, Theoretical aspects of the behavior of beams in accelerators and storage rings, Proceedings of the 1st International School of Particle Accelerators "Ettore Majorana", Erice, Italy, 1976, 1st International School of Particle Accelerators "Ettore Majorana" (CERN, Geneva, 1977).

[7] K. Ochs, A comprehensive analytical solution of the nonlinear pendulum, Eur. J. Phys. 32, 479 (2011).

[8] M. Abramowitz and I. Stegun, Handbook of mathematical functions with formulas, graphs, and mathematical tables, Natl. Bur. Stand. (U.S.), Appl. Math. Ser. 55, 446 (1964).

[9] K. Lasocha and D. Alves, Estimation of longitudinal bunch characteristics in the LHC using Schottky-based diagnostics, Phys. Rev. Accel. Beams 23, 062803 (2020).

[10] A. Beléndez, E. Arribas, M. Ortuño, S. Gallego, A. Márquez, and I. Pascual, Approximate solutions for the nonlinear pendulum equation using a rational harmonic representation, Comput. Math. Appl. 64, 1602 (2012).

[11] D. Boussard, Schottky noise and beam transfer function diagnostics, 1995, https://cds.cern.ch/record/302475/files/ p749.pdf.

[12] J. L. Laclare, Bunched beam coherent instabilities, 1987, https://cds.cern.ch/record/611596/files/p264.pdf.

[13] H. Wiedemann, Particle accelerator physics, Springer Nature, 4th ed. (Springer, New York, 2015).

[14] A. Papoulis and S. U. Pillai, Probability, random variables, and stochastic processes, Tata McGraw-Hill Education, 4th ed. (McGraw-Hill, New York, 2002).

[15] R. E. Kalman, A new approach to linear filtering and prediction problems, J. Basic Eng. 82, 35 (1960).

[16] D. Simon, Optimal State Estimation: Kalman, H Infinity, and Nonlinear Approaches (John Wiley \& Sons, New York, 2006).

[17] D. Simon, Kalman filtering with state constraints: A survey of linear and nonlinear algorithms, IET Contr. Theory Appl. 4, 1303 (2010).

[18] J. Rose et al., RF systems for RHIC, in Proceedings of the Particle Accelerator Conference, Dallas, TX, 1995 (IEEE, New York, 1995), Vol. 3, pp. 1669-1671.

[19] M. Yoshii et al., Present status of J-PARC ring rf systems, in Proceedings of the 22nd Particle Accelerator Conference, PAC-2007, Albuquerque, NM (IEEE, New York, 2007), pp. 1511-1513.

[20] J. Repond, M. Schwarz, and E. Shaposhnikova, Mitigation of intensity limitation in the CERN SPS using a double rf system, Int. J. Mod. Phys. A 34, 1942036 (2019).

[21] R. Garoby, RF gymnastics in synchrotrons, arXiv: 1112.3232 .

[22] J. Y. Liu et al., Analytic solution of particle motion in a double rf system, Part. Accel. 49, 221 (1995). 